The Astronomical Journal, 122:611-620, 2001 August

(C) 2001. The American Astronomical Society. All rights reserved. Printed in U.S.A.

\title{
CALTECH FAINT GALAXY REDSHIFT SURVEY. XV. CLASSIFICATIONS OF GALAXIES WITH $0.2<z<1.1$ IN THE HUBBLE DEEP FIELD NORTH AND ITS FLANKING FIELDS ${ }^{1}$
}

\author{
SidNEY VAN DEN BERGH \\ Dominion Astrophysical Observatory, Herzberg Institute of Astrophysics, National Research Council of Canada, 5071 West Saanich Road, \\ Victoria, BC V9E 2E7, Canada; sidney.vandenbergh@nrc.ca \\ JUdiTh G. COHEN \\ Palomar Observatory, Mail Stop 105-24, California Institute of Technology, Pasadena, CA 91125; jlc@astro.caltech.edu \\ AND \\ CHRISTOPHER CRABBE \\ California Institute of Technology, Mail Stop 185-54, Pasadena, CA 91125; crabbe@its.caltech.edu \\ Received 2001 April 15 ; accepted 2001 May 2
}

\begin{abstract}
To circumvent the spatial effects of resolution on galaxy classification, the images of 233 objects of known redshift in the Hubble Deep Field (HDF) and its flanking fields that have redshifts in the range $0.20<z<1.10$ were degraded to the resolution that they would have had if they were all located at a redshift of $z=1.00$. As in Paper XIV of the present series, the effects of shifts in rest wavelength were mitigated by using $R$-band images for the classification of galaxies with $0.2<z<0.6$ and $I$-band images for objects with redshifts $0.6<z<1.1$. A special effort was made to search for bars in distant galaxies. The present data strongly confirm the previous conclusion that the Hubble tuning fork diagram only provides a satisfactory framework for the classification of galaxies with $z<0.3$. More distant disk galaxies are often difficult to shoehorn into the Hubble classification scheme. The paucity of barred spirals and grand-design spirals at large redshifts is confirmed. It is concluded that the morphology of disk galaxies observed at look-back times smaller than 3-4 Gyr differs systematically from that of more distant galaxies viewed at look-back times of 4-8 Gyr. The disks of late-type spirals at $z>0.5$ are seen to be more chaotic than those of their nearer counterparts. Furthermore, the spiral structure in distant early-type spirals appears to be less well developed than it is in nearby early galaxies.

Key words: galaxies: evolution - galaxies: formation — surveys
\end{abstract}

\section{INTRODUCTION}

The Hubble Space Telescope (HST) has, for the first time, allowed us to undertake systematic imaging surveys (Williams et al. 1996) of galaxies at large redshifts. Furthermore, spectra obtained with the W. M. Keck $10 \mathrm{~m}$ telescope (Cohen et al. 2000) have made it possible to determine redshifts (and hence look-back times) for significant numbers of such distant galaxies. In recent papers (van den Bergh et al. 1996, 2000; Binchmann et al. 1998), ${ }^{2}$ it was found that disk galaxies at redshifts $\gtrsim 0.3$ have morphologies that appear to differ systematically from those of nearby galaxies. A question that presents itself quite insistently is, Could the decrease in linear resolution with increasing distance contribute significantly to these apparent systematic changes of morphology with redshift? In an attempt to answer this question, we have degraded the images of all galaxies with redshifts of $0.20-1.00$ to the appearance that they would have had at $z=1.00$. (The images of 20 galaxies with $1.0<z<1.1$ were left unaltered.) As has already been discussed in van den Bergh et al. (2000, hereafter vdB00), all galaxies were classified at similar rest wavelengths by com-

\footnotetext{
${ }^{1}$ Based in part on observations obtained at the W. M. Keck Observatory, which is operated as a scientific partnership among the California Institute of Technology, the University of California, and the National Aeronautics and Space Administration. The Observatory was made possible by the generous financial support of the W. M. Keck Foundation.

${ }^{2}$ Binchmann et al. (1998) classify galaxies with respect to nine fundamental-type standards. One of us (S. v. d. B.) would have classified their Sbc standard as Sb, their Scd standard as Sc I, and their Ir standard (which appears to have a central nucleus) as a spiral.
}

paring $R$-band images of galaxies having $0.20<z<0.60$ with $I$-band images of objects having $0.60<z<1.10$. For statistical purposes, the present data may be compared with the $B$-band images of galaxies with $z \sim 0.0$ that are seen on the Palomar Sky Survey. The redshifts for individual Hubble Deep Field (HDF) plus flanking field (FF) galaxies in the present paper are from Cohen et al. (2000) and Cohen (2001). Since only a single redshift was available for five merging and/or interacting galaxies, the total number of images of galaxies examined (233) is slightly larger than the total number of redshifts (228).

For more detailed references to previous work on the morphology and classification of distant galaxies, the reader is referred to the excellent review by Abraham (1999).

\section{EFFECTS OF RESOLUTION AND NOISE}

"Postage stamp" images of every individual galaxy with $0.20<z<1.00$ were manipulated in both brightness and angular scale to mimic the appearance that they would have had at $z=1.00$. The images of galaxies with $1.00<z<1.10$ were left untouched. For $z<0.6$, the HST F606W images were used, while the $\mathrm{F} 814 \mathrm{~W}$ images were employed for those galaxies in the sample with $z>0.6$. For many of the galaxies in the flanking fields, no F606W images were available. These objects were therefore omitted from the present study.

The angular scale for each galaxy with $z<1.00$ was compressed by a factor $f=$ (observed angular diameter)/ (angular diameter at $z=1.00$ ), using a cosmology with $H_{0}=60 \mathrm{~km} \mathrm{~s}^{-1} \mathrm{Mpc}^{-1}$ and $\Omega_{M}=0.3$. We then corrected the intensity in the $\mathrm{F} 606 \mathrm{~W}$ images to take into account the 
wavelength dependence of the difference in photon detection efficiency between the WFPC2 instrument behind the F606W and F814W filters. Finally, each image with $z<1.00$ was dimmed by a factor $S=\left[D_{L}(z=1) / D_{L}(z)\right]^{2}$. Furthermore, we took into account the dependence on rest wavelength of the spectral energy distribution of a galaxy; such objects are typically much brighter at $7500 \AA$ in the rest frame than they are at $4050 \AA$.

The procedure outlined above also suppresses the noise in the subimage arising from instrumental contributions and from the sky by the same factor $S$, which for low redshifts may be quite significant. At this point, the contrast between the galaxy and the background is artificially enhanced. To restore the proper noise level, a set of images consisting of random realizations of the noise characteristics of the F606W images in the three wide-field CCDs and in the planetary camera were generated. These were derived from the noise properties measured in the original HST images in regions apparently free of galaxies. Then, for galaxies with $z<0.6$, these were added appropriately to the compressed and rescaled images. ${ }^{3}$ This then creates a set of postage stamp images in which each galaxy appears as it would if that object were situated at $z=1.0$ in the image in which it was originally observed.

Comparison of the new images with those used in vdB00 showed no cases in which our original classifications had to be revised. The only (quite subtle) difference was that faint, possibly tidal, features in the outer parts of some images were more difficult to see than they had been in the original images. No significant differences were seen between the morphologies of the main bodies of our program galaxies before and after an appropriate amount of noise had been added. It is therefore concluded that the classifications of $\mathrm{vdB} 00$ are robust with regard to reasonable changes in the noise level.

\section{CLASSIFICATION OF THE IMAGES}

Table 1 lists the galaxies for which images degraded to $z=1.00$ were available from the survey of Cohen et al. (2000), which was augmented in Cohen (2001). In an attempt to search for weak bars, these images were scrutinized over a larger dynamical range than had previously been explored for the classifications given in vdB00. Classifications for all objects were made by S. v.d.B. and are given on the DDO system (van den Bergh 1960a, 1960b, 1960c). This system is close to that adopted by Sandage \& Tammann (1981). The main difficulty encountered during the classification process was that a significant fraction of the galaxies at large redshifts look "odd" and were therefore often difficult (or impossible) to shoehorn into the classification bins of the Hubble system. For such objects, "pec" (peculiar) was added to their classifications. Since spiral structure in distant galaxies, which are viewed at large look-back times, is less developed than it is in nearby galaxies, the morphology of spiral arms often cannot be used as a classification parameter. Central concentration of light was therefore often used as the primary classification criterion for determining where a spiral galaxy is located on the $\mathrm{Sa}-\mathrm{Sb}-\mathrm{Sc}$ sequence. Inspection of the present images,

\footnotetext{
${ }^{3}$ In principle, this should have been done for the postage stamps with $0.6<z<1.0$ as well, but tests on the lower redshift subimages showed that restoring the correct noise level had no significant effect for our purposes.
}

which were degraded to their appearance at $z=1.00$, strengthens and confirms the previous conclusion (van den Bergh et al. 1996, 2000) that the morphology of late-type disk galaxies at large look-back times is more chaotic than that of nearer spirals of types Sbc-Sc. In distant early-type disk galaxies, the spiral arms are seen to be less developed than they are in nearby $\mathrm{Sa}-\mathrm{Sb}$ galaxies. Since the images used to derive this conclusion had all been degraded to $z=1.0$, the observed decrease in the strength of spiral structure with increasing $z$ cannot be a resolution effect.

The surface density of galaxy images at the depth of the HST images of the HDF and FF is quite high. As a result, these fields contain many apparent close pairs of galaxies, several of which were shown in our previous paper to have widely discrepant redshifts and hence are established as chance projections. Because of this high probability of finding chance pairs, we have, whenever practicable, used tidal deformation of images as a criterion for assigning objects to the "merger" class. Because we have been more conservative in designating objects to this class, the fraction of "mergers" is smaller than it was in vdB00.

Our work provided many examples of late-type galaxies at high redshifts that appeared to be in a very early evolutionary state. With growing experience, we have therefore become more confident in assigning objects to the "protoSc" class. This classification type is therefore now more common than it was in our previous paper. As a result, some objects previously dubbed "Sc pec" have now been reclassified as proto-Sc.

The fact that galaxies at large redshifts appear so odd and are difficult to fit into the Hubble scheme adds significantly to the uncertainty of assigned Hubble types. It is therefore a source of satisfaction to find such good agreement between our original classifications of individual galaxies in vdB00 and the present classifications of images of these same objects degraded to $z=1.0$. A blind comparison between the present new classifications of 99 objects that could be placed on the Hubble sequence E-Sa-Sb-Sc-Ir and those that were previously published for these same objects in vdB00 shows exact agreement for 49 objects, a difference of 0.5 Hubble class for 23 objects, a difference of 1.0 Hubble class for 23 objects, and a difference of greater than 1.0 Hubble class for four objects. For three of these four discrepant classifications, the cause appears to have been that a small fraction of the images classified in vdB00 were not inspected over a sufficiently large dynamic range.

For this sample of 99 galaxies that could be placed on the Hubble sequence E-Sa-Sb-Sc-Ir, there is thus no evidence for any systematic difference in Hubble type between the old classifications and the new classifications of images degraded to $z=1.0$. Furthermore, there is no evidence for any systematic dependence of the differences between the old and new classifications on redshift. Among 56 galaxies with $0.20<z<0.80$, the mean difference, in this sense the old type minus the new, is found to be $-0.04 \pm 0.11$ Hubble classes. For the 41 galaxies on the Hubble sequence with $0.80<z<1.10$, the mean difference, in this sense the old type minus the new, is $-0.08 \pm 0.12$ Hubble classes. Furthermore, the differences between old and new classification types does not appear to depend in a systematic way on Hubble type. On the basis of these results, it is concluded that there are no significant differences between Hubble types assigned in vdB00 and those of degraded images in the present study. A tabulation of the frequencies 
TABLE 1

Classifications of Galaxies

\begin{tabular}{|c|c|c|c|c|}
\hline $\mathrm{ID}^{\mathrm{a}}$ & Redshift $^{\mathrm{b}}$ & $\begin{array}{c}R^{\mathrm{c}} \\
(\mathrm{mag})\end{array}$ & Classification & Comments \\
\hline F36438_1357 ..... & 0.201 & 20.65 & $\mathrm{Sb}:$ & On edge of image \\
\hline F36510_0938 ...... & 0.205 & 21.27 & Ir & Edge-on \\
\hline F36545_1014 ...... & 0.224 & 23.86 & $\mathrm{Sa}$ & \\
\hline H36560_1329..... & 0.271 & 23.80 & $\mathrm{Ir}$ & \\
\hline H36580_1300...... & 0.320 & 22.04 & Sab pec (t?) & Double nucleus \\
\hline F36563_1209 ...... & 0.321 & 23.22 & $\mathrm{Sb} / \mathrm{Ir}$ & Edge-on \\
\hline H36470_1236...... & 0.321 & 20.62 & Sb: pec & \\
\hline H36587_1252..... & 0.321 & 20.99 & $\mathrm{SBc}$ pec & One long arm \\
\hline H36508_1255..... & 0.321 & 22.27 & S pec & Edge-on \\
\hline H36551_1311...... & 0.321 & 23.58 & Merger? & \\
\hline F36458_1325 ...... & 0.321 & 20.71 & Sc pec & Rudimentary spiral structure \\
\hline H36526_1219..... & 0.401 & 23.11 & $\mathrm{E} 4 \mathrm{pec}$ & Embedded in asymmetric nebulosity \\
\hline H36516_1220..... & 0.401 & 21.45 & Sab pec & Binary nucleus \\
\hline F36410_0949 ...... & 0.410 & 20.38 & Ir/merger? & \\
\hline H36472_1230..... & 0.421 & 22.63 & $\mathrm{~S}(\mathrm{~B}$ ?)b pec & Edge-on \\
\hline H36419_1205..... & 0.432 & 20.82 & Sc pec & \\
\hline H36513_1420...... & 0.439 & 23.22 & $\mathrm{Sb}(?)$ pec & \\
\hline F36454_1325 ...... & 0.441 & 22.33 & Sa pec & Asymmetric \\
\hline H36465_1203...... & 0.454 & 24.32 & ? & Tidal debris? \\
\hline H36429_1216..... & 0.454 & 20.51 & Sc I: & \\
\hline H36448_1200...... & 0.457 & 22.85 & Sbc pec & Rudimentary spiral structure \\
\hline H36519_1209...... & 0.458 & 22.75 & $\mathrm{~S}$ pec $(\mathrm{t} ?)$ & \\
\hline H36594_1221...... & 0.472 & 23.53 & $\mathrm{Sa}+\mathrm{S}$ pec & Spectrum probably refers to combined light \\
\hline H36501_1239...... & 0.474 & 20.43 & Sc? pec & Binary nucleus \\
\hline H36569_1302..... & 0.474 & 23.69 & $\mathrm{E} 0 /$ star & \\
\hline H36496_1257...... & 0.475 & 21.91 & Pec & Compact and asymmetric \\
\hline H36572_1259..... & 0.475 & 21.07 & $\mathrm{~S}(\mathrm{~B}) \mathrm{c}$ pec & Perhaps proto-SBc? \\
\hline H36497_1313..... & 0.475 & 21.46 & $\mathrm{Sb}$ pec & Asymmetric \\
\hline H36480_1309...... & 0.476 & 20.43 & E0 pec & Asymmetric envelope \\
\hline H36493_1311...... & 0.477 & 21.97 & $\mathrm{E} 2$ & \\
\hline H36415_1200..... & 0.483 & 25.03 & Pec & \\
\hline H36508_1251..... & 0.485 & 23.15 & Proto-Sc & \\
\hline F36446_1304 ...... & 0.485 & 21.14 & $\mathrm{Sb}$ pec & Proto-Sb? \\
\hline F36427_1306 ...... & 0.485 & 22.02 & $\mathrm{Sab}$ & Edge-on \\
\hline H36528_1404...... & 0.498 & 23.45 & Pec/merger & \\
\hline H36465_1151...... & 0.503 & 22.00 & E0 & \\
\hline F36397_1009 ...... & 0.509 & 21.59 & $\mathrm{Sb}$ pec & Asymmetric \\
\hline H36549_1314..... & 0.511 & 23.81 & $?$ & Edge-on \\
\hline H36489_1245...... & 0.512 & 23.48 & Ir: & \\
\hline H36536_1417..... & 0.517 & 23.36 & $\mathrm{Sab} / \mathrm{S} 0$ & Edge-on \\
\hline F36516_1052 ...... & 0.518 & 23.04 & $\mathrm{Ir}$ & \\
\hline H36566_1245..... & 0.518 & 20.06 & $\mathrm{Sb}$ pec & Smooth disk with little star formation \\
\hline F36213_1417 ...... & 0.520 & 20.73 & $\mathrm{Sa}$ & \\
\hline H36569_1258..... & 0.520 & 23.84 & $\mathrm{E} 5 / \mathrm{Sa}$ & \\
\hline F36425_1518 ...... & 0.533 & 21.79 & Sbc & \\
\hline H36414_1142...... & 0.548 & 23.51 & Merger & Merger of Sa pec + Sa pec $+?+\mathrm{Sab}:$ \\
\hline F36429_1030 ...... & 0.551 & 21.82 & $\mathrm{E}+\mathrm{E}$ merger? & \\
\hline H36442_1247..... & 0.555 & 21.40 & Sc: pec & \\
\hline H36439_1250..... & 0.557 & 20.84 & Pec/merger? & \\
\hline H36517_1353...... & 0.557 & 21.08 & S pec & \\
\hline H36452_1142..... & 0.558 & 24.00 & “Tadpole" & \\
\hline H36555_1359...... & 0.559 & 23.74 & Sbc(pec?) & Edge-on, asymmetric? \\
\hline H36519_1400...... & 0.559 & 23.03 & Sbc pec & Edge-on, asymmetric \\
\hline H36534_1234..... & 0.560 & 22.78 & $\mathrm{Sb} / \mathrm{Ir}$ & Multiple nuclei \\
\hline H36571_1225...... & 0.561 & 22.36 & Sc pec & One-armed spiral \\
\hline H37005_1234..... & 0.563 & 21.43 & E0 & \\
\hline H36554_1402..... & 0.564 & 23.08 & $\mathrm{Sc} / \mathrm{Ir}$ & Edge-on \\
\hline H36413_1141...... & 0.585 & 21.91 & $\mathrm{Sa}$ & \\
\hline H36389_1219...... & 0.609 & 22.14 & Pec & \\
\hline H36471_1414...... & 0.609 & 23.92 & $\mathrm{E} 5+\mathrm{E} 1$ & Spectrum probably refers to combined light \\
\hline F36244_1454 ...... & 0.628 & 20.34 & E2 & \\
\hline F36249_1252 ...... & 0.631 & 22.76 & $\mathrm{Sab} / \mathrm{S} 0$ & \\
\hline F36384_1312 ..... & 0.635 & 22.27 & Sab pec & Asymmetric \\
\hline F37163_1432 ..... & 0.635 & 22.50 & $\mathrm{Sb}$ pec & Asymmetric \\
\hline
\end{tabular}


TABLE 1-Continued

\begin{tabular}{|c|c|c|c|c|}
\hline $\mathrm{ID}^{\mathrm{a}}$ & Redshift $^{\mathrm{b}}$ & $\begin{array}{c}R^{\mathrm{c}} \\
(\mathrm{mag})\end{array}$ & Classification & Comments \\
\hline F36287_1357 ...... & 0.639 & 23.05 & Sa: & \\
\hline F36247_1510...... & 0.641 & 20.41 & $\mathrm{Sab}$ & Rudimentary spiral structure \\
\hline H36538_1254...... & 0.642 & 20.95 & (Proto?)Sc & \\
\hline F36248_1438 ...... & 0.642 & 21.59 & E1 & \\
\hline F36254_1519 ..... & 0.642 & 21.82 & $\mathrm{Sb}$ pec & Asymmetric \\
\hline F36481_1102 ...... & 0.650 & 22.58 & $\mathrm{Sb}$ & \\
\hline F37080_1246 ...... & 0.654 & 21.80 & Sa pec & Asymmetric \\
\hline F36250_1341 ...... & 0.654 & 24.32 & E0/star & \\
\hline F37072_1214 ..... & 0.655 & 22.19 & $\mathrm{Sab}$ & \\
\hline F37213_1120..... & 0.656 & 22.22 & $\mathrm{~S}(\mathrm{~B} ?) \mathrm{c}$ & \\
\hline F37060_1340 ...... & 0.672 & 23.25 & S: & \\
\hline F37171_1122 ...... & 0.676 & 22.98 & E1/star & \\
\hline H36471_1213 ...... & 0.677 & 24.63 & $\mathrm{Sa} \mathrm{pec}+\mathrm{Sa} / \mathrm{E} 3$ & Asymmetric envelope, spectrum probably refers to combined light \\
\hline F36588_1434 ...... & 0.678 & 20.85 & Sb: & \\
\hline H36459_1201...... & 0.679 & 23.88 & S: pec & Edge-on \\
\hline H36502_1245 ...... & 0.680 & 21.74 & $\mathrm{E} 3 / \mathrm{S} 0$ & \\
\hline F36362_1319 ...... & 0.680 & 22.20 & Sb: pec & \\
\hline F36580_1137 ...... & 0.681 & 23.00 & E1 & \\
\hline H36475_1252...... & 0.681 & 24.26 & $\mathrm{Sb}$ pec & Asymmetric \\
\hline F36481_1002 ...... & 0.682 & 21.92 & $\mathrm{Sb}$ pec & \\
\hline H36586_1221...... & 0.682 & 23.40 & E3 pec & Nonelliptical isophotes \\
\hline F36278_1449 ..... & 0.680 & 21.64 & $\mathrm{Sa}$ & \\
\hline F36243_1525 ...... & 0.682 & 22.78 & $\mathrm{E} 2 / \mathrm{Sa}$ & \\
\hline F36454_1523 ...... & 0.683 & 22.06 & E4 & \\
\hline F36499_1058..... & 0.684 & 22.63 & S pec & Arms underdeveloped \\
\hline F37113_1545 ....... & 0.692 & 22.43 & Ir & \\
\hline F37069_1208 ...... & 0.693 & 24.13 & $\mathrm{E} 0 / \mathrm{Sa}$ & \\
\hline F36290_1346 ...... & 0.693 & 23.02 & $\mathrm{E} 2$ & \\
\hline F36427_1503 ...... & 0.698 & 23.17 & $\mathrm{Ir} / \mathrm{pec}$ & Edge-on \\
\hline F36415_0902 ...... & 0.713 & 22.31 & Sc: & \\
\hline F37017_1143..... & 0.744 & 22.10 & $\mathrm{Sa}$ & \\
\hline F37020_1517 ...... & 0.744 & 23.56 & $\mathrm{Sb}$ : & \\
\hline F37036_1353 ..... & 0.745 & 21.63 & $\mathrm{Sb}:$ & \\
\hline F37108_1059..... & 0.747 & 24.25 & $?+?$ & Combined spectrum of two LSB objects \\
\hline F36297_1329 ...... & 0.748 & 23.03 & S: pec & Edge-on \\
\hline F36522_0957 ...... & 0.750 & 23.07 & $\mathrm{Sab}$ pec & Asymmetric \\
\hline H36498_1242........ & 0.751 & 24.38 & Ir & Edge-on \\
\hline F36275_1418 ...... & 0.751 & 22.37 & $\mathrm{Sb}$ & Edge-on, spectrum might refer to Ir? companion \\
\hline H36436_1218...... & 0.752 & 22.56 & $\mathrm{Sa}$ & \\
\hline F37074_1356 ...... & 0.752 & 23.65 & $\mathrm{E} 2 / \mathrm{Sa}$ & \\
\hline H36494_1406...... & 0.752 & 21.95 & $\mathrm{Sa}$ & \\
\hline F37058_1317 ...... & 0.753 & 21.95 & Sc: pec & No spiral arms \\
\hline H36487_1318...... & 0.753 & 22.87 & Proto-Sc & \\
\hline F37061_1332 ...... & 0.753 & 21.85 & $\mathrm{Sb}$ pec & No spiral arms \\
\hline F36297_1324 ...... & 0.758 & 23.25 & Sa pec & Binary nucleus? \\
\hline F36299_1440...... & 0.762 & 22.81 & Sa: pec & \\
\hline F36598_1449 ...... & 0.762 & 21.62 & Merger & \\
\hline H36438_1142...... & 0.765 & 21.26 & $\mathrm{E} / \mathrm{Sa}$ & Asymmetric envelope \\
\hline F36379_0922 ........ & 0.767 & 21.43 & $\mathrm{~S}(\mathrm{~B} ?) \mathrm{b}(\mathrm{pec} ?)$ & Earliest phase of bar formation? \\
\hline F37115_1042 ...... & 0.778 & 21.97 & Sbc pec & \\
\hline F37015_1129..... & 0.779 & 21.45 & Pec/merger & Binary nucleus? \\
\hline F37192_1143 ...... & 0.784 & 22.81 & $\mathrm{Sbc}$ & \\
\hline F37083_1320...... & 0.785 & 22.86 & "Tadpole" & \\
\hline F37222_1124 ...... & 0.786 & 22.33 & $\mathrm{Sa}$ & \\
\hline F37088_1214 ....... & 0.788 & 23.90 & E0/Star & \\
\hline F37105_1141 ...... & 0.789 & 21.20 & Sbc pec & No spiral arms \\
\hline H36555_1245...... & 0.790 & 23.08 & Sbc pec & Rudimentary spiral structure \\
\hline F36299_1403 ...... & 0.793 & 21.97 & Merger & Merger of E0 $t+?$ \\
\hline F36270_1509 ...... & 0.794 & 21.60 & E2 & \\
\hline F36194_1428 ...... & 0.798 & 22.60 & $\mathrm{Pec}$ & \\
\hline F37007_1107 ........ & 0.801 & 22.94 & Sab: & \\
\hline F36175_1402 ...... & 0.818 & 21.73 & Pec & \\
\hline H36503_1418...... & 0.819 & 23.41 & $\mathrm{Sb}$ pec & \\
\hline F37167_1042 ...... & 0.821 & 21.59 & $\mathrm{Ir} /$ merger & \\
\hline F37141_1044 ...... & 0.821 & 22.32 & E2 & \\
\hline F37083_1252 ...... & 0.838 & 22.20 & $\mathrm{Sb}$ pec & No spiral arms, spectrum may include $\mathrm{Sb}$ : companion \\
\hline
\end{tabular}


TABLE 1-Continued

\begin{tabular}{|c|c|c|c|c|}
\hline $\mathrm{ID}^{\mathrm{a}}$ & Redshift $^{\mathrm{b}}$ & $\begin{array}{c}R^{\mathrm{c}} \\
(\mathrm{mag})\end{array}$ & Classification & Comments \\
\hline F37083_1514 ...... & 0.839 & 21.62 & S pec & \\
\hline F37065_1512 ..... & 0.840 & 22.94 & $\mathrm{E} 0 /$ star & \\
\hline F37064_1518 ...... & 0.840 & 22.22 & $?$ & Asymmetric \\
\hline F37105_1116...... & 0.841 & 23.60 & $\mathrm{Sb}(\mathrm{pec} ?)$ & \\
\hline F36447_1455 ...... & 0.845 & 22.97 & $\mathrm{Pec}$ & \\
\hline F36417_0943 ...... & 0.845 & 22.51 & $\mathrm{Sb}$ & \\
\hline F36425_1121..... & 0.845 & 23.03 & $\mathrm{Sb}$ pec & \\
\hline F36343_1312 ........ & 0.845 & 23.15 & Pec/merger? & \\
\hline F36336_1319 ...... & 0.845 & 21.78 & Sc pec & No spiral arms \\
\hline F36420_1321 ...... & 0.846 & 23.95 & Sa pec & Asymmetric envelope \\
\hline F36341_1305 ...... & 0.847 & 24.24 & $\mathrm{Sb}:$ & \\
\hline F36398_1249 ...... & 0.848 & 21.53 & Sa: & Nucleus too small for class \\
\hline F36176_1408 ...... & 0.848 & 22.55 & S pec & \\
\hline H36431_1242...... & 0.849 & 22.34 & $\mathrm{E} 2$ & \\
\hline F36570_1511 ..... & 0.849 & 23.50 & E1: & \\
\hline F36541_1514 ...... & 0.849 & 22.84 & Pec & \\
\hline H36504_1315...... & 0.851 & 23.41 & Sb pec & \\
\hline H36540_1354...... & 0.851 & 22.72 & Sc? & \\
\hline F36462_1527 ..... & 0.851 & 22.11 & $\mathrm{Ir}$ & \\
\hline F36539_1606 ..... & 0.851 & 22.84 & Pec & Nonelliptical isophotes \\
\hline F36589_1208 ...... & 0.853 & 22.32 & $\mathrm{Sb}$ pec & Off-center nucleus \\
\hline F37114_1054 ...... & 0.855 & 22.44 & $\mathrm{Sb}$ & \\
\hline F37089_1202 ..... & 0.855 & 22.90 & Sc pec & Knots but no arms \\
\hline F37129_1028 ..... & 0.858 & 22.62 & Pec: & Edge-on \\
\hline F37096_1055 ...... & 0.858 & 23.16 & $\mathrm{Pec}$ & \\
\hline F37041_1239 ...... & 0.861 & 23.16 & Pec & \\
\hline F36472_1628 ..... & 0.873 & 21.69 & $\mathrm{Sa}$ & \\
\hline F36408_1054 ..... & 0.875 & 22.61 & Merger? & \\
\hline H36441_1240...... & 0.875 & 23.39 & $\mathrm{Pec}$ & \\
\hline F36287_1239..... & 0.880 & 22.11 & Sa: & \\
\hline H36408_1205...... & 0.882 & 22.94 & E1 pec & Asymmetric envelope \\
\hline F36482_1507 ..... & 0.890 & 22.38 & $\mathrm{Sa}$ & \\
\hline F37029_1427 ..... & 0.898 & 23.67 & $\mathrm{E}: 1$ & \\
\hline H36461_1246...... & 0.900 & 22.86 & E1 & \\
\hline F37058_1153 ...... & 0.904 & 21.22 & Sc pec & \\
\hline H36386_1233...... & 0.904 & 24.04 & $\mathrm{Sab}$ & Edge-on \\
\hline F36469_0906 ...... & 0.905 & 23.84 & $\mathrm{Sb}$ pec & Asymmetric, image too small to classify with confidence \\
\hline H36501_1216...... & 0.905 & 23.06 & Proto-Sbc? & Edge-on \\
\hline F37176_1113 ...... & 0.906 & 22.01 & $\mathrm{E} 3 / \mathrm{Sa}$ & \\
\hline F37086_1128 ...... & 0.907 & 22.23 & Merger & \\
\hline F37196_1256 ...... & 0.909 & 23.31 & $\mathrm{Sab}$ & Asymmetric \\
\hline F37180_1248 ...... & 0.912 & 22.89 & $\mathrm{Sbc}$ & \\
\hline F36468_1540...... & 0.912 & 22.23 & Sc(pec?) & \\
\hline F37003_1616...... & 0.913 & 22.33 & Merger? & \\
\hline F37001_1615 ...... & 0.914 & 22.83 & $\mathrm{Sab}$ & \\
\hline F36518_1125 ...... & 0.919 & 21.62 & $\mathrm{Ir} /$ merger? & \\
\hline H36566_1220...... & 0.930 & 23.15 & E2 pec & \\
\hline F37133_1054 ...... & 0.936 & 21.87 & E2 pec & Asymmetric \\
\hline F36522_1537 ..... & 0.936 & 22.74 & E0/star & \\
\hline F36459_1101 ...... & 0.936 & 22.77 & E0 & \\
\hline F37078_1605 ........ & 0.936 & 21.88 & Proto-Sc & \\
\hline F36444_1052 ...... & 0.937 & 23.50 & “Tadpole?" & Might also be an asymmetric Sab \\
\hline F37018_1509 ...... & 0.938 & 22.20 & $\mathrm{Sb}$ pec & \\
\hline F36532_1116 ...... & 0.942 & 22.08 & Proto-Sc? & \\
\hline F36529_1508 ...... & 0.942 & 22.84 & E0/star & \\
\hline H36396_1230...... & 0.943 & 24.40 & $\mathrm{E} / \mathrm{Sa}(\mathrm{pec} ?)$ & \\
\hline H36384_1231...... & 0.944 & 22.87 & Amorphous & Edge-on, off center nucleus? \\
\hline F36465_1049 ...... & 0.945 & 23.70 & $\mathrm{Sa} ?$ & \\
\hline H36555_1249...... & 0.950 & 23.53 & Proto-Sc & \\
\hline H36551_1303...... & 0.952 & 24.29 & E3 & \\
\hline H36576_1315...... & 0.952 & 22.94 & Pec/merger & \\
\hline H36490_1221 ...... & 0.953 & 22.59 & $\mathrm{Pec}$ & Nucleus plus four bright knots \\
\hline F36524_0919..... & 0.954 & 22.81 & E3 & \\
\hline F36502_1127 ..... & 0.954 & 22.88 & Sbc pec & \\
\hline F36520_1059 ...... & 0.955 & 23.67 & $\mathrm{~S}$ & \\
\hline
\end{tabular}


TABLE $1-$ Continued

\begin{tabular}{|c|c|c|c|c|}
\hline $\mathrm{ID}^{\mathrm{a}}$ & Redshift $^{\text {b }}$ & $\begin{array}{c}R^{\mathrm{c}} \\
(\mathrm{mag})\end{array}$ & Classification & Comments \\
\hline H36486_1328...... & 0.958 & 23.14 & Pec/merger & \\
\hline H36477_1232...... & 0.960 & 23.80 & $\mathrm{Sa}$ & \\
\hline F36366_1346 ........ & 0.960 & 20.32 & E0 & \\
\hline H36492_1148...... & 0.961 & 23.26 & $\mathrm{Sa}:$ pec & Asymmetric \\
\hline H36493_1155...... & 0.961 & 23.36 & $\mathrm{E} 2$ & \\
\hline F36364_1237 ...... & 0.961 & 22.94 & $\mathrm{Sb}$ pec & \\
\hline F36486_1141 ...... & 0.962 & 22.21 & $\mathrm{Sb} \mathrm{t}$ & \\
\hline H36483_1214...... & 0.962 & 23.87 & $\mathrm{~S}(\mathrm{~B}) \mathrm{bc} \mathrm{t}$ & \\
\hline H36463_1404...... & 0.962 & 21.69 & E0 & Embedded in large shell \\
\hline F37224_1216 ...... & 0.963 & 22.23 & $\mathrm{Sb}(\mathrm{t} ?)$ & Part of multiple interacting system \\
\hline H36554_1310..... & 0.968 & 22.86 & E1 & \\
\hline F37058_1423 ...... & 0.970 & 22.48 & Sb: & \\
\hline F37055_1129 ...... & 1.001 & 22.37 & Sa pec & \\
\hline H36432_1148...... & 1.010 & 23.10 & $\mathrm{Sb}$ pec & Smooth disk with little star formation? \\
\hline H36408_1203...... & 1.010 & 23.49 & Ir & Edge-on \\
\hline H36461_1142......... & 1.013 & 21.52 & Proto-Sc & \\
\hline F37000_1605 ...... & 1.013 & 22.46 & Sc: & On edge of image \\
\hline F37154_1212 ...... & 1.014 & 23.25 & E pec & \\
\hline H36400_1207...... & 1.015 & 22.75 & E0 & \\
\hline F36411_1314 ....... & 1.017 & 23.08 & Sa pec & Asymmetric \\
\hline F36497_1106 ....... & 1.018 & 23.37 & $\mathrm{Pec}$ & \\
\hline H36444_1142...... & 1.020 & 24.30 & $\mathrm{Pec} /$ merger & \\
\hline F37159_1213 ..... & 1.020 & 23.27 & Sbc pec & \\
\hline F36583_1214 ...... & 1.020 & 23.79 & E2 & \\
\hline F36595_1153 ...... & 1.021 & 22.54 & Sa pec $(t ?)$ & Asymmetric \\
\hline H36443_1133...... & 1.050 & 21.96 & E1 & \\
\hline F37046_1415 ..... & 1.050 & 23.97 & Sab: & \\
\hline F36296_1420 ...... & 1.055 & 24.00 & E0/star & \\
\hline H36467_1144....... & 1.060 & 24.23 & Proto-Sc & \\
\hline F37026_1216 ...... & 1.073 & 24.04 & Sab pec & Asymmetric \\
\hline F37143_1221 ...... & 1.084 & 24.12 & $\mathrm{E}: 2$ & Image too small to classify with confidence \\
\hline H36519_1332....... & 1.087 & 23.59 & Sa pec & \\
\hline
\end{tabular}

of various classification types of galaxies in Table 1 as a function of redshift is shown in Table 2.

\section{GALAXY STATISTICS}

\subsection{Frequency of Barred Galaxies}

It was first noted that barred spirals appear to be deficient among galaxies at high redshift by van den Bergh et al. (1996). Subsequently, this shortage of barred objects at $z>0.5$ was confirmed (in both the northern and southern HDF) by Abraham et al. (1999). Bothun (2000) suspected that the absence of SB galaxies at high redshifts might have been due to band-shift effects, which result from the fact that bars appear to be more frequent in the $H$-band (Eskridge et al. 2000) than they are in the $B$-band. However, the observations of bar frequencies at various redshifts by vdB00, which were obtained at an almost constant rest wavelength, appear to rule out this explanation.

TABLE 2

FREQUENCY Distribution OF GALAXY TyPeS

\begin{tabular}{crrccclcccc}
\hline \hline$z$ & $n(z)$ & $\mathrm{E}^{\mathrm{a}}$ & $\mathrm{Sa}+\mathrm{Sab}$ & $\mathrm{Sb}+\mathrm{Sbc}^{\mathrm{b}}$ & $\mathrm{Sc}+\mathrm{Sc} / \mathrm{Ir}^{\mathrm{b}}$ & $\mathrm{Ir}$ & $\mathrm{Pec}$ & Pec/Merger & Merger & $?$ \\
\hline $0.20-0.29 \ldots \ldots$ & 4 & 0 & 1 & 1 & 0 & 2 & 0 & 0 & 0 & 0 \\
$0.30-0.39 \ldots \ldots$ & 7 & 0 & 1 & 2.5 & 1 & 0.5 & 1 & 0 & 1 & 0 \\
$0.40-0.49 \ldots \ldots$ & 25 & 4 & 4 & 5 & $4+(1)$ & 0 & 2 & 1 & 0 & 0 \\
$0.50-0.59 \ldots \ldots$ & 24 & 5 & 2 & 5 & 3 & 2.5 & 0 & 1 & 1 & 1 \\
$0.60-0.69 \ldots \ldots$ & 37 & 14 & 7 & 7 & $1+(1)$ & 1.5 & 1 & 0 & 0 & 0 \\
$0.70-0.79 \ldots \ldots$ & 32 & 4 & 7 & 8 & $2+(1)$ & 1 & 1 & 1 & 2.5 & 2 \\
$0.80-0.89 \ldots \ldots$ & 41 & 7 & 7 & $9+(1)$ & 3 & 1.5 & 9 & 1 & 2 \\
$0.90-0.99 \ldots \ldots$ & 43 & 14 & 5 & 11 & $2+(2)$ & 0.5 & 1 & 2 & 2.5 & 0 \\
$1.00-1.09 \ldots \ldots$ & 20 & 6 & 6 & 0 & $1+(1)$ & 1 & 1 & 1 & 0 & 0 \\
Total ........ & 233 & 55 & 40 & $48.5+(1)$ & $17+(6)$ & 10.5 & 16 & 7 & 9 & 4 \\
\hline
\end{tabular}

${ }^{a}$ Includes E, E0/Star, E/Sa, S0, and S0/Sa.

b Numbers in parentheses refer to objects that were classified as proto-Sb or proto-Sc. 
In the present investigation, special attention was paid to the possible presence of bars by inspecting each program galaxy over the widest possible dynamic range. Out of 233 images, only a single one was found to be a pure barred spiral of type SB, while one was an intermediate-type object classified as S(B). Furthermore, three galaxies were classified $\mathrm{S}(\mathrm{B}$ ?). The corresponding percentages are $0.4 \% \mathrm{SB}$, $0.4 \% \mathrm{~S}(\mathrm{~B})$, and $1.3 \% \mathrm{~S}(\mathrm{~B}$ ?), respectively. For comparison, Sandage \& Tammann (1981) found that $22 \%$ of all ShapleyAmes galaxies that have $z \sim 0.0$ are barred. These data suggest that the fraction of all galaxies that are barred is at least an order of magnitude lower in the distant HDF plus FF sample than it is in the nearby Shapley-Ames sample. It is noted in passing that the only two certain barred galaxies in the present sample [which were classified SB and S(B)] have $z<0.5$. Nevertheless, it is puzzling that the fraction of barred spirals at all redshifts appears to be so much lower on HST images than it is on photographs of nearby galaxies. Simulations to investigate this problem are currently being undertaken by Abraham \& van den Bergh (2001).

Since the universe is expanding, galaxies were once closer together than they are at the present time. As a result, tidal interactions and mergers are expected to be more frequent at high redshifts than they are at the present time. Evidence based on galaxy morphology in favor of this view was first provided by observations of galaxies in the Hubble Deep Field (van den Bergh et al. 1996). It is possible that the high frequency of tidal interactions in the early universe also contributed to the shortage of large, well-developed disks in galaxies at $z \gg 1$, which was already noted above. Using the present (rather strict) definition of mergers, the data in Table 2 show that 11 out of $116(9 \%)$ of all galaxies with $0.70<z<1.00$ are classified as either "merger" or "peculiar/merger." From inspection of the entire data set, one of us (S. v.d.B.) has the impression that the rate of multiple mergers may be increasing faster with redshift than the rate of binary mergers.

\subsection{Changes in the Relative Frequencies of Galaxy Types as a Function of Redshift}

Table 2 shows the frequency distributions of different morphological types in the present sample as a function of redshift. The data in this table may be compared to those of the northern Shapley-Ames galaxies (van den Bergh 1960c) given in vdB00. The Shapley-Ames galaxies that are located at $z \sim 0.0$ were classified on reproductions of the $B$ images of the Palomar Sky Survey. They have therefore been observed at approximately the same rest wavelength as the $H S T$ images of galaxies with $z>0.20$. Since galaxies of types $\mathrm{E}, \mathrm{Sa}$, and $\mathrm{Sb}$ have rather similar luminosity func-

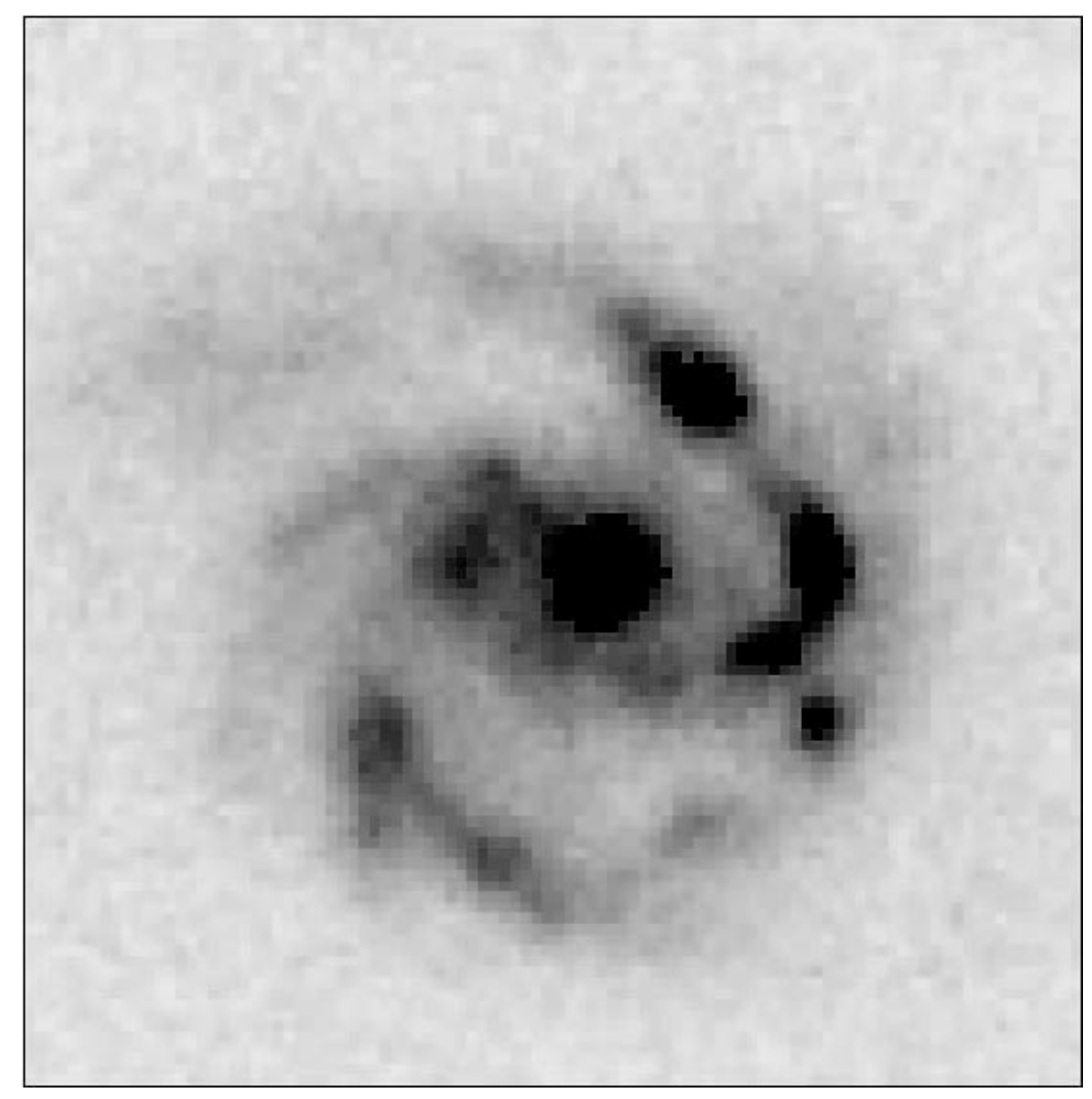

FIG. 1.-Example of what is interpreted as a proto-Sc galaxy. Note the rather chaotic spiral structure and very patchy arm morphology of H36461_1142 at $z=1.01$. 


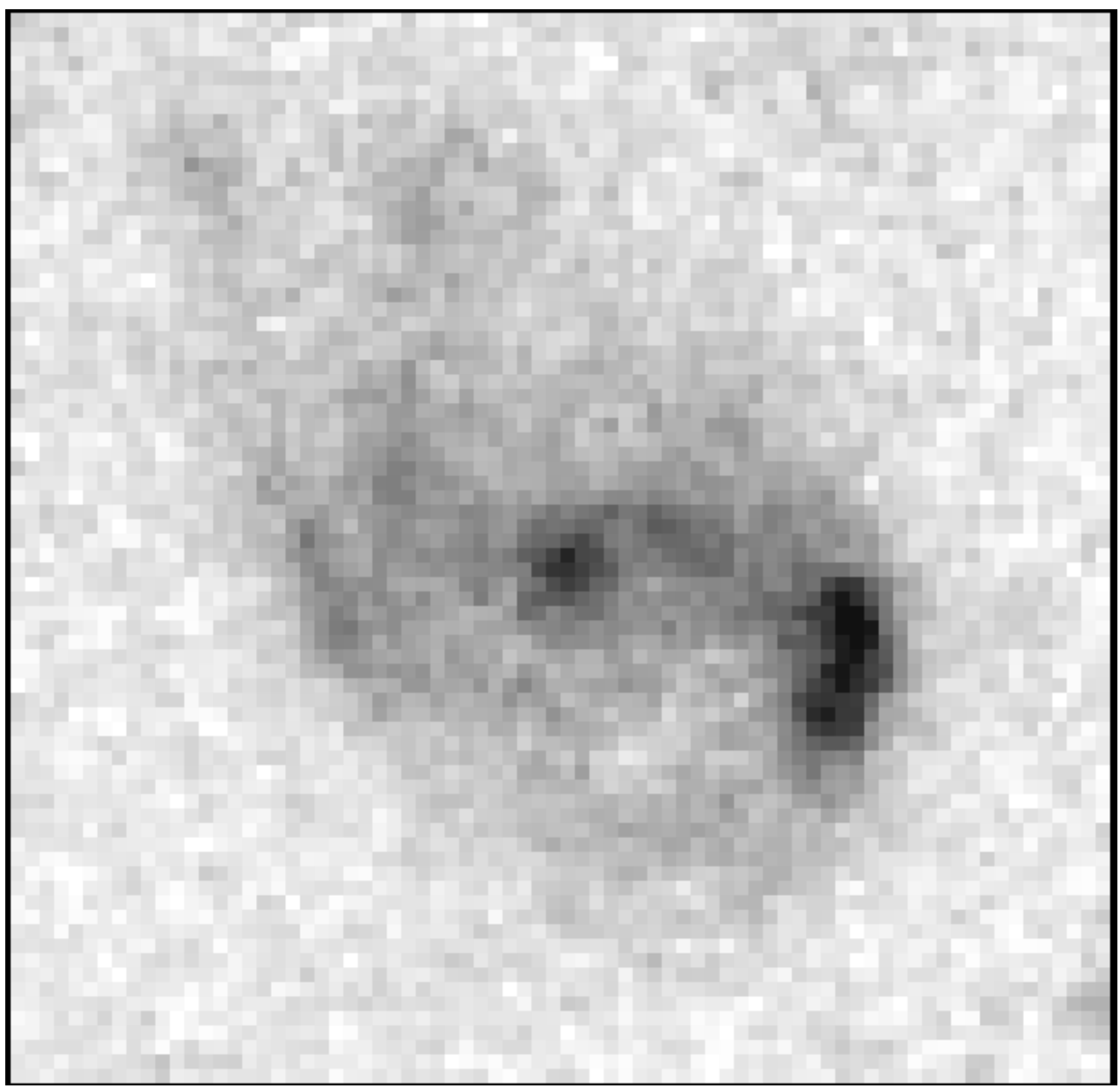

FIG. 2.-Image of a spiral(?) galaxy (H36467_1144) that might be interpreted as either a proto-Sc with a very patchy arm structure or the product of a recent merger. Kinematical studies of this object at $z=1.06$ will be required to distinguish between these two alternatives.

tions, their relative frequencies (as a function of redshift) should not be strongly affected by redshift-dependent selection effects. However, the present luminosities of late-type spirals are, on average, significantly lower than those of galaxies having earlier Hubble types (see Fig. 1 of van den Bergh 1998). In the absence of evolutionary effects, highredshift samples of galaxies might therefore be biased against objects of types Sc and Ir. It should, however, be emphasized that this bias against late-type galaxies will be lessened (or might even be reversed) if such objects exhibit significant luminosity evolution. Lilly et al. (1995) and Cohen (2000) have, for example, demonstrated that galaxies with $0.5<z<0.75$ might have brightened by as much as $1 \mathrm{mag}$. On the other hand, Carollo \& Lilly (2001) have more recently noted that the high metallicity of late-type galaxies with $0.5<z<1.0$ makes it improbable that these objects are luminosity-enhanced dwarfs.
The data in Table 2 suggest that the fraction of late-type $(\mathrm{Sc}+\mathrm{Sc} / \mathrm{Ir}+\mathrm{Ir})$ galaxies decreases with increasing redshift. For the redshift bins $z=0.20-0.49,0.50-0.79$, and 0.80 1.09 , the percentage of late-type galaxies is found to be $19 \%$, $12 \%$, and $8 \%$, respectively. Taken at face value, this trend suggests that the fraction of late-type galaxies decreases with increasing redshift. Such an effect could, if real, be explained by assuming that a large fraction of all Sc galaxies have not yet been fully assembled or are still classified as proto-Sc at $z>0.5$. Alternatively, if luminosity evolution is unimportant, this effect might be due to a distancedependent selection effect that results from the fact that late-type galaxies are (on average) less luminous than earlytype galaxies (see Fig. 1 of van den Bergh 1998).

The present sample is so small that bins containing objects in a small range of redshifts and morphological types provide pitifully small statistical samples of galaxies. 


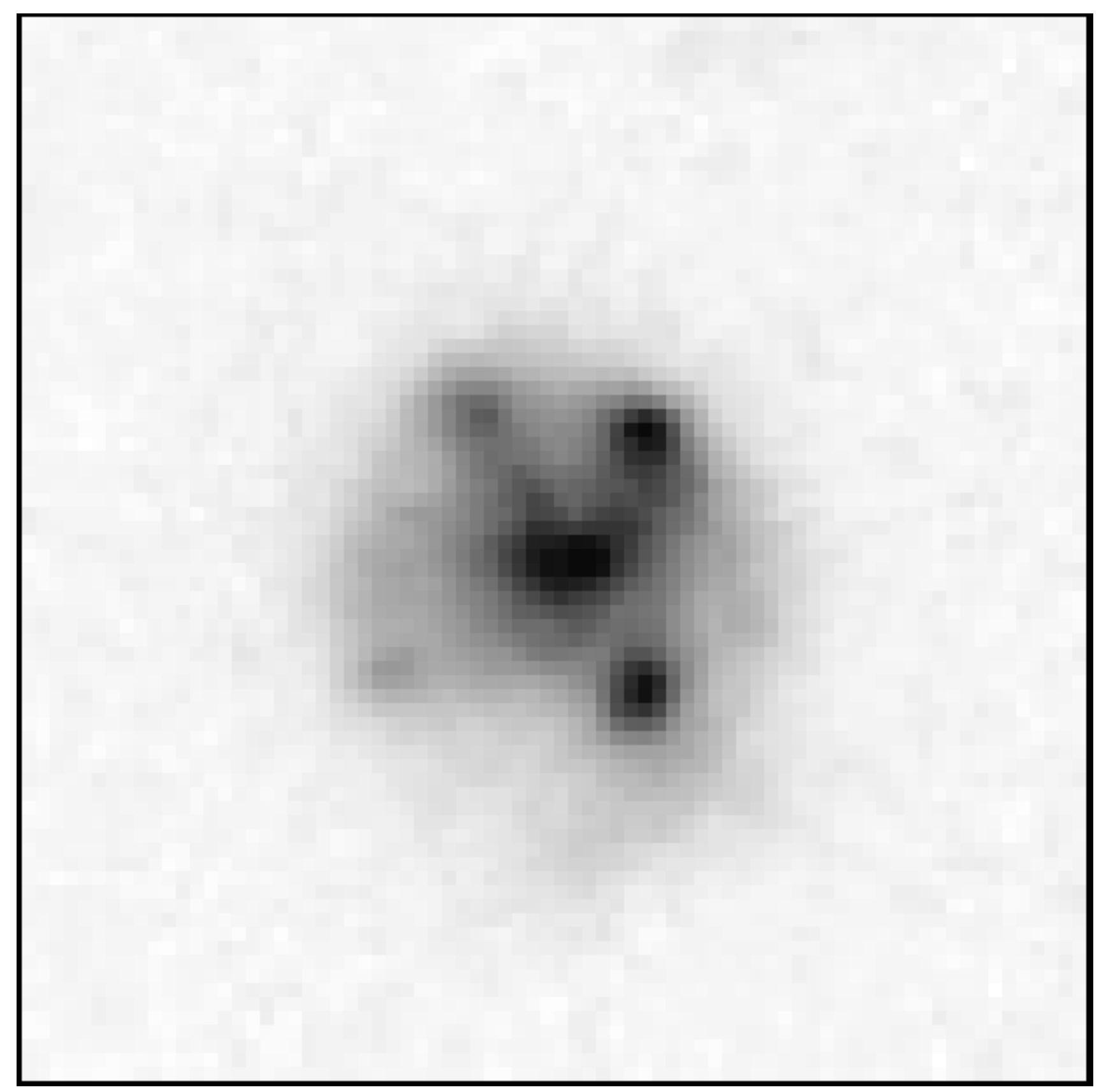

Fig. 3.-Peculiar galaxy (H36490_1221) at $z=0.953$ that consists of a disk in which five bright knots, one of which might be the nucleus, are embedded. No spiral structure appears to have developed yet in this object.

Furthermore, the distribution of morphologies at a given redshift might be affected by density enhancements as the line of sight passes through sheets or clusters of galaxies. Intersection of the line of sight toward the HDF with a populous group of galaxies at $z \sim 0.68$ is, for example, responsible for an excess of E galaxies in the $z=0.60-0.69$ redshift bin. As a result of the vagaries of small number statistics and the effects of local density enhancements on galaxy morphology, it would be unwise to draw very strong conclusions from the present data about the morphological evolution of galaxies over time.

The apparent increase in the number of elliptical galaxies with $z>0.9$ might be spurious, even if it is not due to the vagaries of small number statistics. This is so because any small compact high surface brightness object with regular oval isophotes will be classified as an "elliptical galaxy." This includes the class of galaxies denoted as "blue compact galaxies" (see, e.g., Phillips et al. 1997; Guzmán et al. 1997), a local example of which might be NGC 6789. These two groups are not easily distinguished morphologically in this redshift regime. However, from the spectra and SEDs of the set of galaxies under consideration here given in earlier papers in this series, we are confident that many of these galaxies are not classical elliptical galaxies, but are instead strongly star-forming blue galaxies that are compact.

\section{MORPHOLOGICAL EVOLUTION OF GALAXIES}

Since the majority of elliptical galaxies are believed to have formed at $z>1.0$, the present galaxy sample is not expected to contain many proto-elliptical galaxies. Never- 
theless, some compact pairs and groupings of early-type galaxies might, in the fullness of time, merge into run-of-themill elliptical galaxies. A few $\mathrm{E}$ and Sa galaxies in the present sample exhibit faint chaotic outer structures that might have resulted from such earlier mergers.

Many of the early-type ( $\mathrm{Sa}, \mathrm{Sab}$ ) spirals in our sample exhibit morphological peculiarities. The most common of these are (1) underdeveloped spiral structure, (2) warped disks, and (3) off-center nuclear bulges. A large fraction of the late-type (Sbc, Sc, and $\mathrm{Sc} / \mathrm{Ir}$ ) galaxies in our sample of high-redshift galaxies exhibit morphological peculiarities that distinguish them from their nearby counterparts. The disks of distant Sc galaxies tend to be much more chaotic than those of nearby luminous galaxies, which generally exhibit a well-ordered spiral structure. This effect is particularly significant because the sample of distant late-type galaxies is biased in favor of objects of above-average luminosity. Such galaxies are expected (van den Bergh 1960a) to exhibit particularly long and regular spiral arms. Distant single Sc spirals sometimes have one strongly dominant spiral arm. Among nearby galaxies, such single-arm objects are usually objects that have been tidally perturbed. Furthermore, the disks of proto-Sc galaxies, in which spiral structure is not yet well developed, are often seen to contain luminous blue knots (superassociations). In other words, huge localized bursts of star formation appear to occur before a well-ordered spiral pattern develops. If the regions in which such superassociations occur have significant over densities, they could experience strong dynamical friction and spiral toward the galactic center (Noguchi 1998), where they might contribute to the formation of a galactic bulge.

Figure 1 shows a good example of an Sc I galaxy at $z=1.01$ that is just beginning to form well-ordered spiral structure. Note that the arms of this object are much patchier and more chaotic than those of typical nearby Sc I galaxies. The luminous patches (superassociations) in this object are seen to be much more luminous than those that occur in typical Sc I galaxies at $z \sim 0.0$. A color image of an even more primitive late-type galaxy is shown in Figure 5 (Plate 9) of van den Bergh et al. (1996). This object exhibits what appears to be a disk in which 10 bright blue knots (superassociations) are embedded. A single slightly offcenter red knot may be the (slightly older) nuclear bulge of this probable protogalaxy. Figure 2 shows an example of an object at $z=1.06$ that might be a proto-Sc with a single arm that contains a superassociation. Alternatively, this object might be interpreted as the final phase of a merger. Spectroscopic observations will be required to distinguish between these two alternatives. Finally, Figure 3 shows what may be another example of a protogalactic disk in which a nucleus and four other bright knots appear to be embedded.

\section{CONCLUSIONS}

The present data have allowed us to study the evolution of galaxy morphology over the range $0.2<z<1.1$ at an effective wavelength that is insensitive to redshift and at a spatial resolution that is independent of $z$. The main conclusion that can be drawn from this work is that the morphology of disk galaxies has evolved rapidly over the last $8 \mathrm{Gyr}$. At look-back times greater than $4 \mathrm{Gyr}$, late-type spirals generally have a much more chaotic structure than do nearby galaxies of types Sbc and Sc. Furthermore, the arms of such distant spirals tend to be patchy and are also often asymmetric. At look-back times greater than $4 \mathrm{Gyr}$, earlytype spiral galaxies appear to have less well-developed spiral structure than do their counterparts, which are viewed at look-back times of less than 3-4 Gyr. Apparently, insufficient time was available for spiral structure to develop in such early-type galaxies. Among distant galaxies in the Hubble Deep Field, the intrinsic frequency of barred spirals appears to be at least an order of magnitude lower than it is for nearby galaxies at $z \sim 0$. It is concluded that the Hubble (1936) tuning fork scheme is only appropriate for the classification of galaxies at $z<0.3$ that are viewed at look-back times of less than 3-4 Gyr.

We are indebted to Roger Blandford and David Hogg for their assistance and advice during the course of the present investigation. We also thank Bob Abraham for his help with the figures.

\section{REFERENCES}

Abraham, R. G. 1999, Ap\&SS, 269-270, 323

Abraham, R. G., Merrifield, M. R., Ellis, R. S., Tanvir, N. R., \& Brinchmann, J. 1999, MNRAS, 308, 569

Abraham, R. G., \& van den Bergh, S. 2001, in preparation

Binchmann, J., et al. 1998, ApJ, 499, 112

Bothun, G. D. 2000, S\&T, 99(5), 36

Carollo, C. M., \& Lilly, S. J. 2001, ApJ, 548, L153

Cohen, J. G. 2000, in Deep Fields, ed. S. Cristiani, A. Renzini, \& R. E.

Williams (New York: Springer), in press (astro-ph/0012004)

. 2001, AJ, 121, 2895

Cohen, J. G., Hogg, D. W., Blandford, R. D., Cowie, L. L., Hu, E., Songaila,

A., Shopbell, P., \& Richberg, K. 2000, ApJ, 538, 29

Eskridge, P. B., et al. 2000, AJ, 119, 536

Guzmán, R., Gallego, J., Koo, D. C., Phillips, A. C., Lowenthal, J. D., Faber, S. M., Illingworth, G. D., \& Vogt, N. P. 1997, ApJ, 489, 559

Hogg, D. W., et al. 2000, ApJS, 127, 1

Hubble, E. 1936, The Realm of the Nebulae (New Haven: Yale Univ. Press), 45
Lilly, S. J., Tresse, L., Hammer, F., Crampton, D., \& Le Févre, O. 1995, ApJ, 455, 108

Noguchi, M. 1998, Nature, 392, 253

Phillips, A. C., Guzmán, R., Gallego, J., Doo, D. C., Lowenthal, J. D., Vogt,

N. P., Faber, S. M., \& Illingworth, S. D. 1997, ApJ, 489, 543

Sandage, A., \& Tammann, G. A. 1981, A Revised Shapley-Ames Catalog of Bright Galaxies (Washington: Carnegie Inst.)

van den Bergh, S. 1960a, ApJ, 131, 215

. 1960b, ApJ, 131, 558

1960c, Publ. David Dunlap Obs., 2, 159

1998, Galaxy Morphology and Classification (Cambridge: Cambridge Univ. Press)

van den Bergh, S., Abraham, R. G., Ellis, R. S., Tanvir, N. R., Santiago,

B. X., \& Glazebrook, K. G. 1996, AJ, 112, 359

van den Bergh, S., Cohen, J. G., Hogg, D. W., \& Blandford, R. 2000, AJ, 120,2190

Williams, R. E., et al. 1996, AJ, 112, 1335 